Tokyo etabliert das erste städtische $\mathrm{CO}_{2}$-Emissionshandelssystem

\section{Klimaschutz lokal}

\author{
Während Japan im internationalen Klimaschutz derzeit \\ auf der Bremse steht, geht Tokyo einen innovativen \\ Weg. Seit dem Jahr 2010 läuft dort das erste städtische \\ $\mathrm{CO}_{2}$-Emissionshandelssystem. Die ersten Erfahrungen \\ zeigen Stärken und Schwächen. \\ Von Sven Rudolph und Takeshi Kawakatsu
}

G roßstädte sind weltweit bereits heute für 70 Prozent der energiebedingten Emissionen von Kohlenstoffdioxid $\left(\mathrm{CO}_{2}\right)$ verantwortlich, Tendenz steigend. Tokyo, die weltgrößte Metropolregion und fünfzehntgrößte Volkswirtschaft, verursacht allein ein Niveau vergleichbar mit nordeuropäischen Ländern.

95 Prozent dieser Emissionen sind energiebedingte $\mathrm{CO}_{2}$-Emissionen, wobei Tokyos Pro-Kopf-Emissionen mit 4,89 Tonnen pro Jahr und seine Kohlenstoffintensität von 146 Kilotonnen pro Milliarde US-Dollar des Bruttoinlandsproduktes gering sind. Gleichwohl stiegen die $\mathrm{CO}_{2}$-Emissionen in Tokyo zwischen 1990 und 2006 um rund sechs Prozent, sinken seitdem aber stetig. Rund die Hälfte der energiebedingten $\mathrm{CO}_{2}$-Emissionen stammen aus wirtschaftlichen Aktivitäten, davon vier Fünftel aus Handel und Dienstleistungen und nur ein Fünftel aus der industriellen Produktion. Von den gesamten $\mathrm{CO}_{2}$-Emissionen aus wirtschaftlichen Aktivitäten werden rund 40 Prozent von Großemittenten verursacht.

\section{Tokyos innovatives Emissionshandelssystem}

Vor diesem Hintergrund setzte sich Tokyo Treibhausgasreduktionsziele von 25 Prozent bis 2020 und 50 Prozent bis 2050 gegenüber dem Jahr 2000 und verabschiedete eine Klimaschutzstrategie. Neben freiwilligen, ordnungsrechtlichen und planerischen Instrumenten wird seit April 2010 das Tokyo Metropolitan Go- vernment Emissions Trading Scheme (TMG ETS) genutzt.

Angesichts der Ausgangslage fokussiert das TMG ETS auf $\mathrm{CO}_{2}$-Emissionen aus großen Gebäuden und Industrieanlagen mit einem Jahresendenergieverbrauch von 1.500 Kilolitern Öläquivalent. Es umfasst damit rund 1.300 Einrichtungen und mit 13 Millionen Tonnen rund 20 Prozent aller $\mathrm{CO}_{2}$-Emissionen. Die Teilnahme ist dabei, anders als bei Japans nationalem Emissionshandelssystem, verpflichtend.

Die Emissionsgesamtmenge, das Cap, wurde auf sechs Prozent beziehungsweise 17 Prozent unter den Basisjahremissionen für die erste Handelsperiode von 2010 bis 2014 und die zweite Handelsperiode von 2015 bis 2019 limitiert, wobei für die erste ein Ziel von minus sechs Prozent für Industrieanlagen und minus acht Prozent für Bürogebäude gilt. Das Basisjahr können betroffene Einrichtungen aus drei aufeinanderfolgenden Jahren zwischen 2002 und 2007 wählen, so dass sich das Gesamtcap aus den individuellen Reduktionsverpflichtungen ergibt. Das Gesamtcap wurde für das Jahr 2020 auf 10,44 Millionen Tonnen $\mathrm{CO}_{2}$ geschätzt.

Das TMG ETS vergibt nicht wie etwa der EU-Emissionshandel echte Emissionslizenzen, sondern definiert individuelle Reduktionsverpflichtungen. Können die Einrichtungen ihre Reduktionsverpflichtung übererfüllen, so erhalten sie Emissionsreduktionsgutschriften, die sie dann verkaufen können. Prinzipiell werden damit Emissionsrechte kostenlos vergeben.
Transaktionen finden derzeit allein bilateral statt, da keine Handelsplattform existiert; Tokyo engagiert sich jedoch selbst in der Zusammenführung von Angebot und Nachfrage. Gehandelt werden dürfen nur zertifizierte Emissionsreduktionsgutschriften, nicht aber, wie in anderen Emissionshandelssystemen, alle zugeteilten Emissionsrechte.

Das Banking von Emissionsreduktionsgutschriften ist möglich, Borrowing jedoch untersagt. Emissionsgutschriften, sogenannte Offsets, aus dem Clean Development Mechanism (CDM) und Joint Implementation (JI) werden nicht akzeptiert, während unter anderem solche aus kleinen und mittelständischen Unternehmen in Tokyo sowie aus ErneuerbareEnergien-Projekten anerkannt werden.

Bei Nicht-Einhaltung der Vorgaben werden Strafen von bis zu 500.000 Yen, das entspricht rund 5.000 Euro, plus eine Nacherfüllung der Reduktionsverpflichtung im Umfang des 1,3-fachen der Überschussemissionen fällig. Das Monitoring basiert dabei auf verifizierten Emissionsberichten.

\section{Gute Ansätze}

Tokyo betritt mit seinem Emissionshandelssystem Neuland. Es etabliert das erste ambitionierte $\mathrm{CO}_{2}$-Cap-and-TradeSystem in Japan, den weltweit ersten städtischen Kohlenstoffmarkt und setzt erstmals bei der Endenergienutzung an. Ähnlich wie in der Pilotphase des EUEmissionshandels lassen sich daher Stärken und Schwächen identifizieren.

Das Design wirkt auf den ersten Blick vielversprechend. Das TMG ETS ist für die betroffenen Einrichtungen verpflichtend und bezieht alle Großemittenten des wichtigsten Treibhausgases $\mathrm{CO}_{2}$ ein. Das Cap wird absolut gesetzt und die Reduktionsziele erscheinen vor dem Hintergrund vergleichbarer Ziele in der EU und den USA anspruchsvoll. Ebenso können das Monitoring als verlässlich und die Strafen als abschreckend gelten. Das erwartete Preisniveau von 100 bis 150 USDollar pro Tonne $\mathrm{CO}_{2}$ liegt so auch beträchtlich höher als in anderen Systemen. Die Akzeptanz von Banking und der Aus- 
schluss von Borrowing sowie die Einbeziehung anspruchsvoller Offsets bei gleichzeitigem Ausschluss von fragwürdigen CDM-Projekten sind ebenfalls positiv zu bewerten.

\section{Gravierende Designfehler}

Kritisch sind jedoch besonders die kostenlose Zuteilung von Emissionsrechten und das Handelssystem. Eine kostenlose Zuteilung verzögert verlässliche Preissignale und kann zu Wettbewerbsverzerrungen führen. Zudem wird kein Aufkommen generiert, das für effizienzsteigernde, redistributive oder klimaschützende Maßnahmen genutzt werden könnte. Die Beschränkung des Handels auf Überschussreduktionen und das Fehlen einer institutionalisierten Handelsplattform behindern eine optimale Allokation von Vermeidungsmaßnahmen. Nicht zuletzt deckt das TMG ETS nur 20 Prozent der $\mathrm{CO}_{2}$-Emissionen ab.

Die Designfehler zeigen sich dann auch in den ersten praktischen Erfahrungen. Zwar erscheinen die Reduktionsziele im internationalen Vergleich als ambitioniert, bereits im Jahr 2009, ein Jahr vor dem Start des Projekts, lagen die Emissionen aber mit minus zehn Prozent unter dem Ziel für die erste Phase. Rund 60 Prozent der betroffenen Einrichtungen übererfüllten schon 2009 ihre Phase-IVerpflichtungen, 22 Prozent sogar ihre Verpflichtungen für Phase II.

\section{Anpassungen notwendig}

Ein aktiver Handel hat sich noch nicht etabliert. So wurde bis April 2012 nur eine Transaktion im Wert von 360 Tonnen $\mathrm{CO}_{2}$ durchgeführt. In 14 Fällen wurden Emissionskredite im Gesamtumfang von rund 31.300 Tonnen $\mathrm{CO}_{2}$ ausgestellt; alle bezogen sich aber auf Offsets, während keine einzige echte Emissionsreduktionsgutschrift ausgestellt wurde. Zudem hatten bis dato nur 200 Einrichtungen ein für den Handel notwendiges Konto eröffnet.

Ein kosteneffizienter Kohlenstoffmarkt mit einem bindenden $\mathrm{CO}_{2}$-Cap und Anreizen zu echten Emissionsmin- derungen über das Business-as-usual hinaus hat sich damit noch nicht etabliert. Insgesamt stellt das TMG ETS aber einen wichtigen und innovativen Schritt zu einem Klimaschutz von unten dar, der als Inspiration für andere Gebietskörperschaften dienen kann. Um die ökologische Effektivität und die ökonomische Effizienz des TMG zu erhöhen, empfehlen sich vor allem eine Verschärfung der Ziele, die schrittweise Einführung einer Auktionierung sowie die Etablierung einer Handelsplattform.

\section{Literatur}

Rudolph, S. / Kawakatsu, T.: Tokyo's Greenhouse Gas Emissions Trading Scheme - A Model for Sustainable Megacity Carbon Markets? MAGKS Joint Discussion Paper Series in Economics No. 25. 2012.

TMG - Tokyo Metropolitan Government: Tokyo Cap-and-Trade Program - Japan's first mandatory emissions trading scheme. Tokyo 2010.

I AUTOREN + KONTAKT

Dr. Sven Rudolph ist wissenschaftlicher Assistent für die Politische Ökonomie der Klimapolitik am Competence Centre for Climate Mitigation and Adaptation (CliMA) der Universität Kassel.

E-Mail: s.rudolph@wirtschaft.uni-kassel.de Takeshi Kawakatsu, PhD, ist Associate Professor an der Faculty of Public Policy der Kyoto Prefectural University.

\section{soziale}

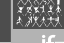
technik

Seit 21 Jahren ist SOZIALE TECHNIK - herausgegeben vom Interuniversitären Forschungszentrum für Technik, Arbeit und Kultur (IFZ) - ein Forum für die interdisziplinäre Technikforschung und liefert Beiträge für eine sozial- und umweltverträgliche Gestaltung von Technologien. SOZIALE TECHNIK erscheint vierteljährlich, ein Jahresabonnement kostet $€ 21,50$ (für Studierende $€ 15,-$ ).

Kostenloses Probeabonnement!

\section{Mit Beiträgen zu:}

Technologie \& Politik

$\square$ Umwelt \& Energie

$\square$ Neue Biotechnologien

- Frauen \& Technik

口 Informations- \&

Kommunikationstechnologien
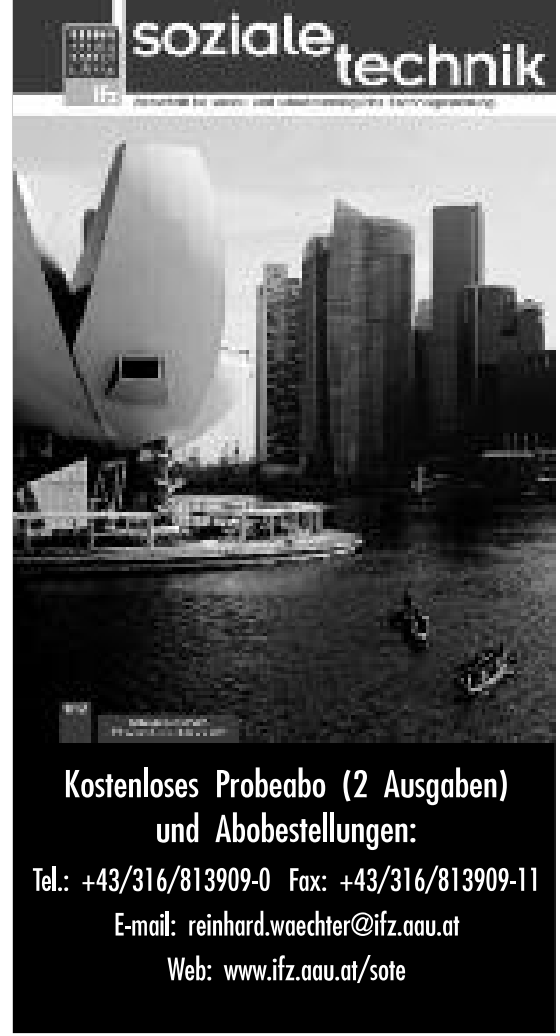


\section{Lizenzhinweis}

Die Beiträge in ÖkologischesWirtschaften werden unter der Creative-Commons-Lizenz "CC 4.0 Attribution Non-Commercial No Derivatives" veröffentlicht. Im Rahmen dieser Lizenz muss der Autor/Urheber stets genannt werden, das Werk darf nicht bearbeitet, abgewandelt oder in anderer Weise verändert und außerdem nicht kommerziell genutzt werden. Die digitale Version des Artikels bleibt für zwei Jahre Abonnent/innen vorbehalten und ist danach im Open Access verfügbar. 